\title{
Galectin-3 and acute heart failure: genetic polymorphisms, plasma level, myocardial fibrosis and 1-year outcomes
}

\author{
Hao Fu' ${ }^{1}$, Shaoping $\mathrm{Ni}^{1}{ }^{1 \mathrm{D}}$, Ping Luo ${ }^{1}$, Yang Ruan ${ }^{3}$, Zichuan Zhang ${ }^{2}$, Huangtai Miao' ${ }^{1}$ Xin \\ $\mathrm{Li}^{2}$ (iD), Songnan Wen² \& Rong Bai*,2,4 (iD \\ ${ }^{1}$ Emergency \& Critical Care Center, Beijing Anzhen Hospital, Capital Medical University, Beijing, China \\ ${ }^{2}$ Department of Cardiology, Beijing Anzhen Hospital, Capital Medical University, Beijing, China \\ ${ }^{3}$ Department of Cardiology, Beijing Tiantan Hospital, Capital Medical University, Beijing, China \\ ${ }^{4}$ Texas Cardiac Arrhythmia Institute at St. David's Medical Center, Austin TX 78706, USA \\ *Author for correspondence: Tel.: +86186101 01790; Fax: +86108400 5361; bairong74@gmail.com
}

\begin{abstract}
Aim: This study sought to investigate the relationship between galectin-3 (Gal-3), myocardial fibrosis (MF) and outcomes in acute heart failure. Materials \& methods: The single-nucleotide polymorphisms (SNPs) of LGALS3 at rs4644 and rs4652, plasma Gal-3 level, MF and major adverse events (MAEs) were obtained. Results: There was no significant difference in MAEs when categorizing patients by the LGALS3 SNPs at rs4644 and rs4652. The circulating Gal-3 was related to the degree of MF ( $p<0.001)$. Plasma Gal-3 level and MF can predict an increased risk of MAEs $(p<0.001, p=0.023$, respectively). Conclusion: Not the SNPs of LGALS3 but Gal-3 and MF can predict MAEs in acute heart failure at 1 year of follow-up.
\end{abstract}

First draft submitted: 5 May 2020; Accepted for publication: 9 June 2020; Published online: 17 September 2020

Keywords: acute heart failure $\bullet$ galectin-3 $\bullet$ major adverse event $\bullet$ myocardial fibrosis $\bullet$ polymorphism

Acute heart failure (AHF) is the advanced and decompensated phase of heart failure (HF) and accounts for the first cardiac cause of hospital admission in the elderly. Patients with AHF usually present with a rapid onset of new or worsening clinical manifestations of HF, and their admission to the hospital predicts a higher rate of re-hospitalization and mortality post discharge. The overall mortality of hospitalized patients with HF is $4.1 \%$ in China [1], while data from the UK have demonstrated that the post discharge 30-day and 1-year mortality rates of patients with AHF are 6.5 and 30\% respectively [2]. It has been well acknowledged that, regardless of etiology, myocardial fibrosis (MF) plays a vital role in the pathogenesis, progression and prognosis of HF, including its acute type. Previous studies have shown that galectin-3 (Gal-3) is a biomarker of tissue fibrosis and its serum level offers significant prognostic value for adverse outcomes in patients with HF [3-6]. Binding the $\beta$-galactosides, Gal-3 belongs to the galectin family and is generated by activated macrophages and fibroblasts [7]. LGALS3 is the Gal-3 coding gene which is located on chromosome 14 (locus q21-q22) [8]. A recent study has indicated that two LGALS3 gene single nucleotide polymorphisms (SNPs) (rs4644 and rs4652) are associated with changes in protein levels [9] and may alter the circulating Gal-3 content, but less is known about these genetic variations in relation to MF or adverse outcome in AHF patients. In the present study, we prospectively investigated the relationship between Gal-3 coding gene polymorphism and circulating plasma Gal-3, MF, and prognosis in a cohort of patients with AHF.

\section{Materials \& methods}

\section{Study population}

From April 2016 to December 2017, we prospectively enrolled 200 consecutive patients diagnosed as AHF with reduced ejection fraction that were hospitalized to the Emergency and Critical Care Center of Beijing Anzhen Hospital, Capital Medical University. The inclusion criteria of our study were: two major or one major and two minor criteria for the Framinghan score for HF [10]; left ventricular ejection fraction (LVEF) $<40 \%$ by echocardiography; 
symptoms were developed or aggravated within 1 week preceding admission; patients aged from 18 to 80 years. Exclusion criteria included: BNP level of less than $100 \mathrm{pg} / \mathrm{ml}$; infiltrative cardiomyopathy; extracardiac fibrotic disease, autoimmune disease and malignant tumor; severe renal dysfunction (eGFR $<30 \mathrm{ml} / \mathrm{min} / 1.73 \mathrm{~m}^{2}$ ); patients with prior implantable cardiac devices and severe claustrophobia, who could not undergo cardiac MRI.

\section{Clinical assessment}

All patients underwent a comprehensive clinical assessment at the time of their admission, including echocardiography, chest $\mathrm{x}$-ray and electrocardiogram. Full medical history data as well as information on current and prior therapies were also obtained. For echocardiographic parameters, left ventricular end-diastolic diameter (LVEDD) was measured from the parasternal long-axis view, and LVEF was calculated by the biplane Simpson method from the apical 4-chamber view [11]. All these echocardiographic measurements were collected within $48 \mathrm{~h}$ after admission. Routine lab tests including BNP, cardiac TNI (cTNI), creatinine, uric acid, electrolytes, plasma albumin, high-sensitivity CRP (hs-CRP) and hemoglobin were performed by the Central Laboratory of Beijing Anzhen Hospital. Specifically, eGFR was calculated with the Modification of Diet in Renal Disease equation [12]. Blood samples for routine lab tests, genotyping and fibrosis biomarker evaluation were collected at the time of admission.

\section{Genotyping of Gal-3 coding gene (LGALS3)}

Within 30 min of collection, blood samples were centrifuged at $1000 \times g$ at $4^{\circ} \mathrm{C}$ for $15 \mathrm{~min}$. Total genomic DNA was extracted and purified using the Puregene Kit (Gentra Systems, MN, USA). All samples were stored at $-80^{\circ} \mathrm{C}$ for future assay. We used the US National Center for Biotechnology Information dbSNP database to extract information on the SNPs in LGALS3. According to a previous study about LGALS3 SNPs [9], we determined two sites (rs4644 and rs4652) to be sequenced in our study by using ABI Prism 3730xl DNA analyzers (Thermo Fisher Scientific, MA, USA) with standard protocols. PCR was performed using HotStarTaq polymerase (Qiagen, Hilden, Germany). The primers used were 5' CTT TCT TTC CAG CTC CAT GA 3' (rs4644-F) and 5' CCC TTG AGA AGT GGC TCT AA 3' (rs4644-R).

\section{Plasma Gal-3 measurement}

The plasma concentrations of Gal-3 and sST2 were measured by an ELISA (R\&D Systems, MN, USA) according to the manufacturer's instructions.

\section{Cardiac MRI}

Each patient underwent standard cardiac MRI scanning (3.0-Tesla Magnetom Verio; Siemens AG Healthcare, Erlangen, Germany) when he/she became clinically stable during the index hospitalization period. Cine magnetic resonance (MR) images were acquired in vertical and horizontal long-axis and a stack of short-axis planes covering the left ventricle. Using a phase-sensitive inversion recovery sequence, late gadolinium enhancement (LGE) imaging was conducted in the same planes at $15 \mathrm{~min}$ following the administration of $0.1 \mathrm{mmol} / \mathrm{Kg}$ of gadopentetate dimeglumine. The LGE technique was applied to quantify MF by using the QMass software (Medis, Leiden, The Netherlands). The LGE-positive region was defined as an area where the signal intensity was six standard deviations higher than the average signal intensity of the healthy myocardial tissue in the background of the noise signal. Left ventricular endo and epicardial outlines were tracked on the short-axis LGE images, which included the total left ventricle from the base to the apex. A region of interest was traced by an operator on an area of unenhanced myocardium, and the total LGE volume was quantified using four semiautomated techniques and compared with manual adjustment results of the signal intensity threshold. According to the method mentioned above, the software used the sum of the products of the volume and the thickness of each layer as the total left ventricle myocardial volume and automatically calculated the percentage of the LGE-positive region in the total left ventricle myocardial volume, expressed as $\mathrm{V}_{\mathrm{LGE}} / \mathrm{V}_{\mathrm{t}} \%$ (LGE positive volume/total left ventricle myocardial volume $\times \%$ ), which represents a quantitative indicator of MF in the left ventricle. We also evaluated left ventricular function measurements including LVEF, stroke volume and cardiac output.

\section{Follow-up}

All patients were followed up with for 1 year after discharge. It was required that every patient presented at Beijing Anzhen Hospital for at least one office visit during this period. Telephone interviews were periodically conducted by the primary investigator. During follow-up, what we recorded included symptoms, changes of medication and 
the study end point, which was defined as major adverse event (MAE) within 1 year including all-cause mortality and re-hospitalization for HF. The censoring time was determined when any of the two events first occurred. If a patient died in or readmitted to our hospital, this information was available from the digital medical records system. If an end point event occurred in another hospital, the information was acquired during the office visit or telephone interview.

\section{Statistical analysis}

We used the Kolmogorov-Smirnov method to test for normal distribution, then continuous data were presented as means and standard deviations, or medians and interquartile ranges. Categorical data were presented as numbers (percentages). The mean values were compared using the Student's $t$-test and the median values were compared using Mann-Whitney $U$ test. The Chi square test was used for comparing categorical variables. The Kruskal-Wallis $\mathrm{H}$ test was used to determine the differences in plasma Gal-3 concentration and severity of MF. Kaplan-Meier curves were plotted to estimate the cumulative event-free survival, of which the difference was assessed by log-rank test.

Due to the observed non-normality distribution, plasma Gal-3 level and quantitative indicator of MF $\mathrm{V}_{\mathrm{LGE}} / \mathrm{V}_{\mathrm{t}} \%$ were log-transformed to $\ln \mathrm{Gal}-3$ and $\ln \left(\mathrm{V}_{\mathrm{LGE}} / \mathrm{V}_{\mathrm{t}} \%+1\right)$, respectively, to achieve normality for Pearson correlation coefficient and linear regression analysis. Cox proportional-hazards regression models were built to identify independent predictors of MAEs. For the Cox regression, cofounders known to have an influence on MAEs or which were relevant to the present study were first entered in a univariate model, and those showed significance $(p<0.1)$ were then entered in a multivariate model. The ascendency of the combination of plasma Gal-3 and MF over plasma Gal-3 alone in predicting MAEs were analyzed by calculating the augmentation in the global chi-square.

All statistical analyses were performed using the Statistical Package for the Social Sciences (SPSS) version 20.0 software program (IBM Corp, NY, USA). A p $<0.05$ was considered statistically significant.

\section{Results}

\section{Baseline characteristics \& MAE}

Overall, 200 patients with AHF with reduced ejection fraction were enrolled, among whom the mean age was $54 \pm 15$ years and $59 \%$ were male. Within 1 year of follow-up, 25 patients (12.5\%) died and 53 patients (26.5\%) were re-hospitalized for HF. Therefore, 58 patients (29\%) reached the study end point of MAE. Comparisons of patients' baseline characteristics between the MAE group and nonevent group are summarized in Tables $1 \& 2$.

Briefly, patients in the MAE group who were generally older ( 57 vs 52 years; $\mathrm{p}=0.036$ ), had a higher prevalence of chronic kidney disease (22.4 vs 8.5\%; $\mathrm{p}=0.007)$ and lower eGFR values (72.97 vs $86.14 \mathrm{ml} / \mathrm{min} / 1.73 \mathrm{~m}^{2} ; \mathrm{p}<$ 0.001). At admission, patients with MAE presented with higher BNP (2567.5 vs $942.5 \mathrm{pg} / \mathrm{ml}$; p < 0.001), cTNI (0.12 vs $0.03 \mathrm{ng} / \mathrm{ml} ; \mathrm{p}=0.001)$ and sST2 (40.12 vs $28.2 \mathrm{ng} / \mathrm{ml} ; \mathrm{p}<0.001)$ levels, as well as greater LVEDD (66.34 vs $62.9 \mathrm{~mm} ; \mathrm{p}=0.009)$ and lower natrium $(137.79$ vs $139.34 \mathrm{mmol} / \mathrm{ml} ; \mathrm{p}=0.01)$, hemoglobin (138.98 vs $146.76 \mathrm{~g} / \mathrm{l} ; \mathrm{p}=0.016)$ levels and LVEF on cardiac MRI (17.4 vs $22.37 \%$; $\mathrm{p}<0.001)$. Further, they required more frequent use of intravenous vasodilators during index hospitalization ( 48.3 vs $31 \%$; $\mathrm{p}=0.021$; Tables $1 \& 2$ ).

\section{LGALS3 SNPS \& GAL-3 \& MF}

In this cohort of Chinese patients, the $\mathrm{C}$ allele frequency of the SNP rs 4644 was $60 \%$ and the A allele frequency was $40 \%$, while the C allele frequency of the SNP rs 4652 was $39.75 \%$ and the A allele frequency was $60.25 \%$. Additionally, the plasma Gal-3 level was $9.97 \mathrm{ng} / \mathrm{ml}$ in the entire study population and significantly higher in those with MAE ( $28.21 \mathrm{vs} 7.99 \mathrm{ng} / \mathrm{ml}$ in nonevent group; $\mathrm{p}<0.001$; Table 2 ), but was not statistically different among the LGALS3 genotyping (i.e., AA, AC, CC) groups of the rs4644 and rs4652 (p = 0.392 and $\mathrm{p}=0.954$, respectively; Figure 1A \& B).

Similarly, patients who developed MAE during follow-up had more severe left ventricle MF indicated as higher $\mathrm{V}_{\mathrm{LGE}} / \mathrm{V}_{\mathrm{t}} \%$ (25.68 vs $6.59 \%$ in nonevent group; $\mathrm{p}<0.001$; Table 2$)$. However, this measurement was comparable in subjects with variant LGALS3 genotypes (i.e., AA, AC, CC) of the rs 4644 and rs4652 ( $\mathrm{p}=0.841$ and $\mathrm{p}=0.65$, respectively; Figure $2 \mathrm{~A} \& \mathrm{~B}$ ). 


\begin{tabular}{|c|c|c|c|}
\hline Baseline characteristics & MAE $(n=58)$ & Nonevent $(n=142)$ & p-value \\
\hline Male gender, $\mathrm{n}(\%)$ & $35(60.3)$ & $83(58.5)$ & 0.805 \\
\hline Age, years $(\text { mean } \pm S D)^{\dagger}$ & $57 \pm 16$ & $52 \pm 15$ & 0.036 \\
\hline BMI, kg/m² (median, IQR) & $23.01(21.13-25.33)$ & $23.6(21.8-26.23)$ & 0.148 \\
\hline \multicolumn{4}{|l|}{ Post medical history, n (\%) } \\
\hline CHD & $29(50)$ & $73(51.4)$ & 0.857 \\
\hline Hypertension & $24(41.4)$ & $72(50.7)$ & 0.231 \\
\hline Diabetes mellitus & $23(39.7)$ & $54(38)$ & 0.830 \\
\hline Dyslipidemia & $28(48.3)$ & $72(50.7)$ & 0.755 \\
\hline $\mathrm{CKD}^{\dagger}$ & $13(22.4)$ & $12(8.5)$ & 0.007 \\
\hline COPD & $2(3.4)$ & $2(1.4)$ & 0.705 \\
\hline SAHS & $0(0)$ & $7(4.9)$ & 0.195 \\
\hline \multicolumn{4}{|l|}{ Medications, n (\%) } \\
\hline IV vasoconstrictors & $3(5.2)$ & $2(1.4)$ & 0.295 \\
\hline IV vasodilators $^{\dagger}$ & $28(48.3)$ & $44(31)$ & 0.021 \\
\hline IV positive inotropic agents & $7(12.1)$ & $20(14.1)$ & 0.705 \\
\hline Diuretics & $58(100)$ & $142(100)$ & - \\
\hline Digitalis & $24(41.4)$ & 46 (32.4) & 0.227 \\
\hline ACEI/ARB & $46(79.3)$ & $124(87.3)$ & 0.150 \\
\hline Beta-blocker & $57(98.3)$ & $138(97.2)$ & 1.000 \\
\hline MRA & $52(89.7)$ & $120(84.5)$ & 0.341 \\
\hline CRT & $1(1.7)$ & $5(3.5)$ & 0.826 \\
\hline ICD & $4(6.9)$ & $8(5.6)$ & 0.990 \\
\hline \multicolumn{4}{|c|}{$\begin{array}{l}\text { Vasoconstrictors included dopamine, epinephrine, noradrenaline; vasodilators included nitrate, nesiritide, nitroprusside sodium; positive inotropic agents included dobutamine, } \\
\text { milrinone, levosimendan. } \\
\text { †The variables of which p-value is less than } 0.05 \text {. } \\
\text { ACEI: Angiotensin converting enzyme inhibitor; ARB: Angiotensin receptor antagonist; CHD: Coronary heart disease; CKD: Chronic kidney disease; COPD: Chronic obstructive } \\
\text { pulmonary disease; CRT: Cardiac resynchronization therapy; ICD: Implantable cardioverter defibrillator; IQR: Interquartile range; IV: Intravenously; MAE: Major adverse event; } \\
\text { MRA: Mineralocorticoid receptor antagonist; SAHS: Sleep apnea hypopnea syndrome; SD: Standard deviation. }\end{array}$} \\
\hline
\end{tabular}
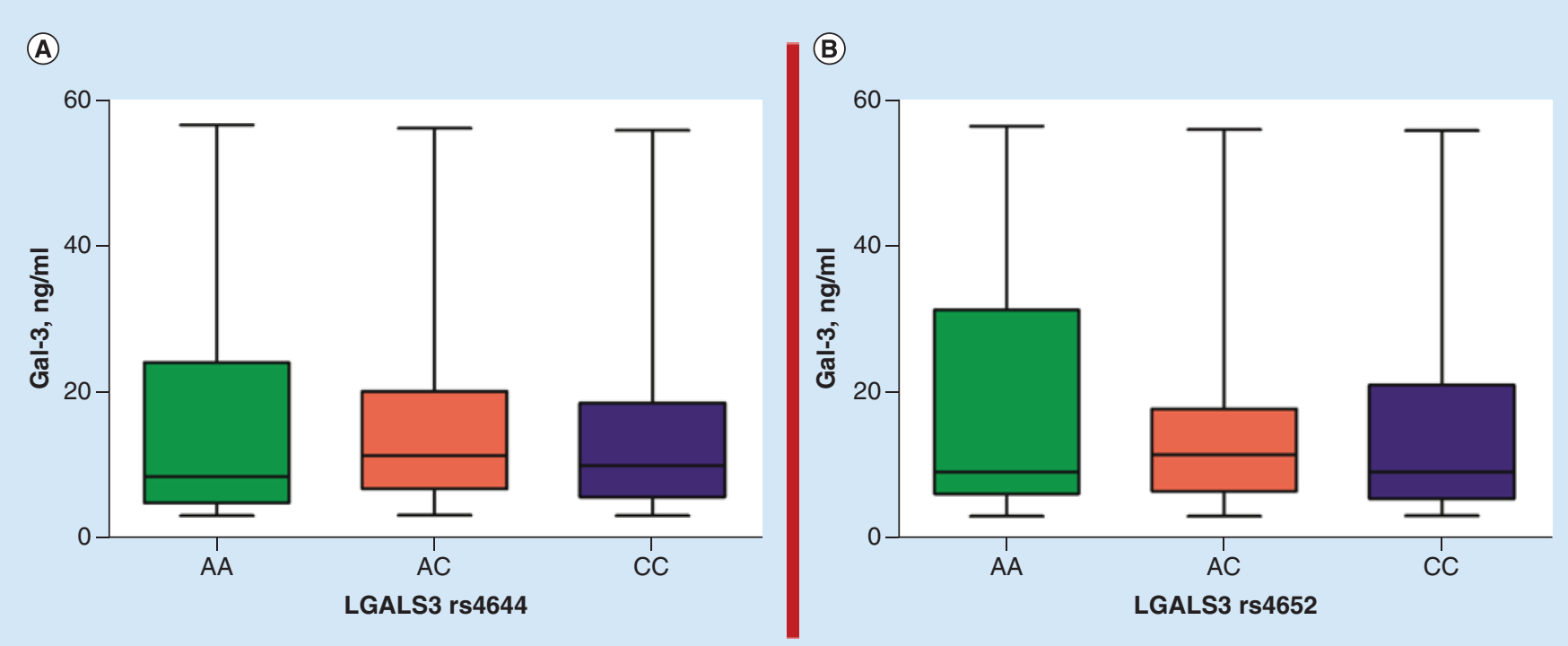

Figure 1. Plasma galectin-3 levels categorized by the LGALS3 single-nucleotide polymorphisms. Plasma Gal-3 levels in patients with the AA, AC and CC genotypes of the SNPs rs4644 (A) and rs4652 (B) were not significantly different $(p=0.392, p=0.954$, respectively). Gal-3: Galectin-3; SNP: Single nucleotide polymorphism. 
Table 2. Clinical indices in patients with and without major adverse event.

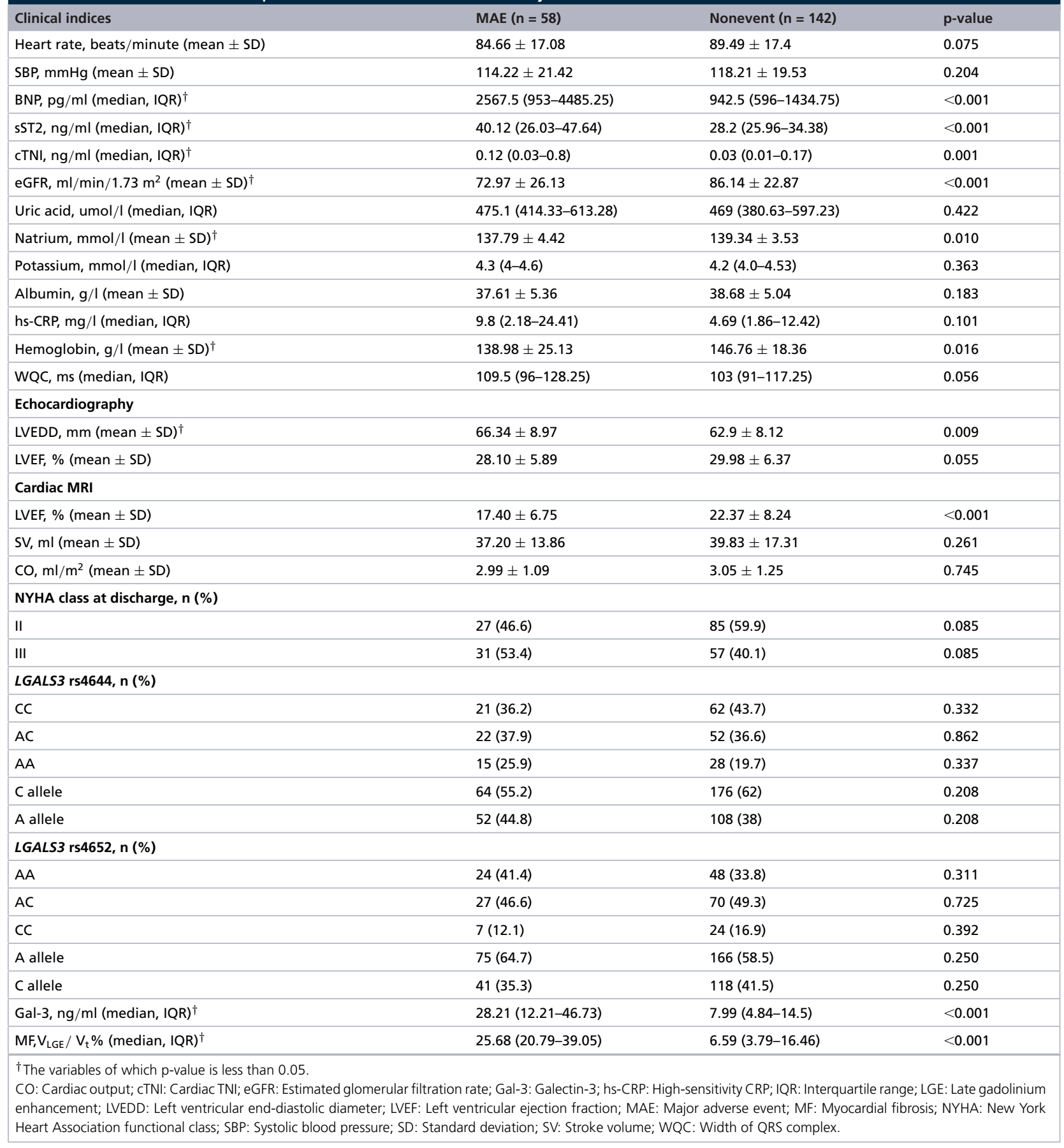

\section{LGALS3 SNPS \& MAE}

During 1 year of follow-up, no significant differences were found between the MAE group and the nonevent group with regard to LGALS3 genotype distribution and allelic frequencies for the SNPs rs4644 and rs4652 ( $\mathrm{p}>0.05$, respectively; Table 2). This was also evident by the results of Kaplan-Meier survival curves according to LGALS3 genotypes (AA, AC and CC) of the SNP rs4644 (log-rank p = 0.393; Figure 3A) and the SNP rs4652 (log-rank p $=0.407$; Figure 3B). 


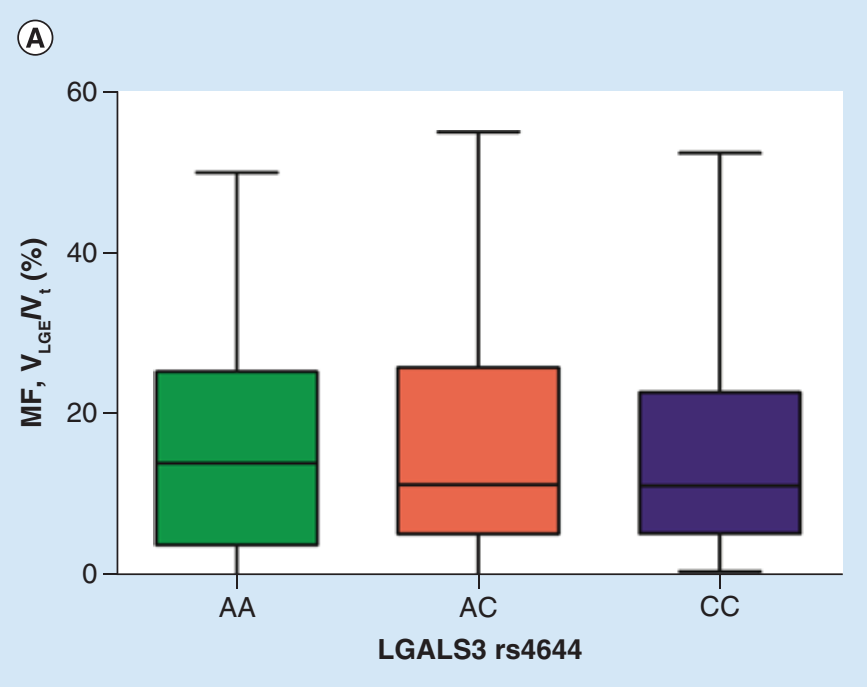

(B)

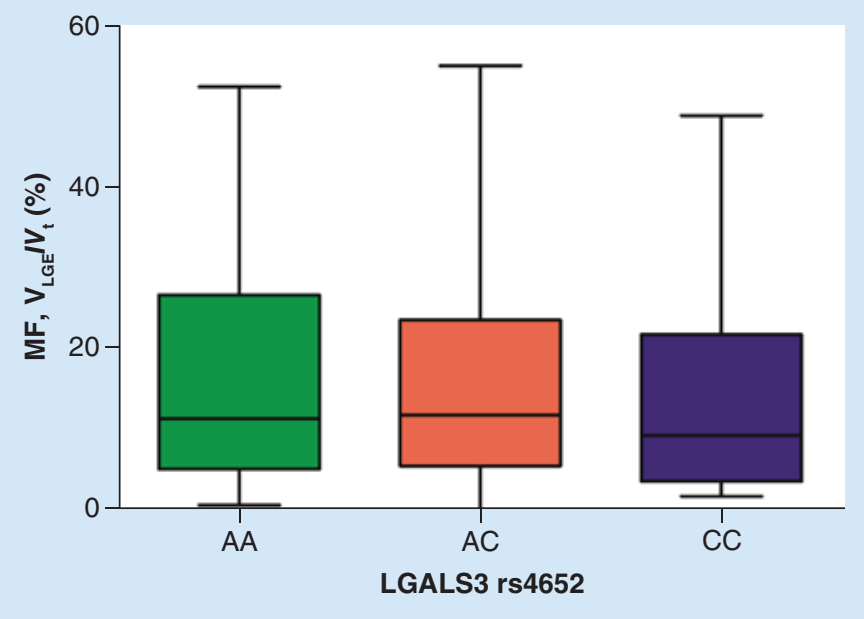

Figure 2. Severity of myocardial fibrosis categorized by the LGALS3 single-nucleotide polymorphisms. MF in patients with the LGALS3 genotypes (AA, AC and CC) of the rs4644 (A) and rs4652 (B) were not significantly different $(p=0.841, p=0.65$, respectively). LGE: Late gadolinium enhancement; MF: Myocardial fibrosis; $V_{L G E}$ : LGE positive volume; $V_{t}$ : Total left ventricle myocardial volume.

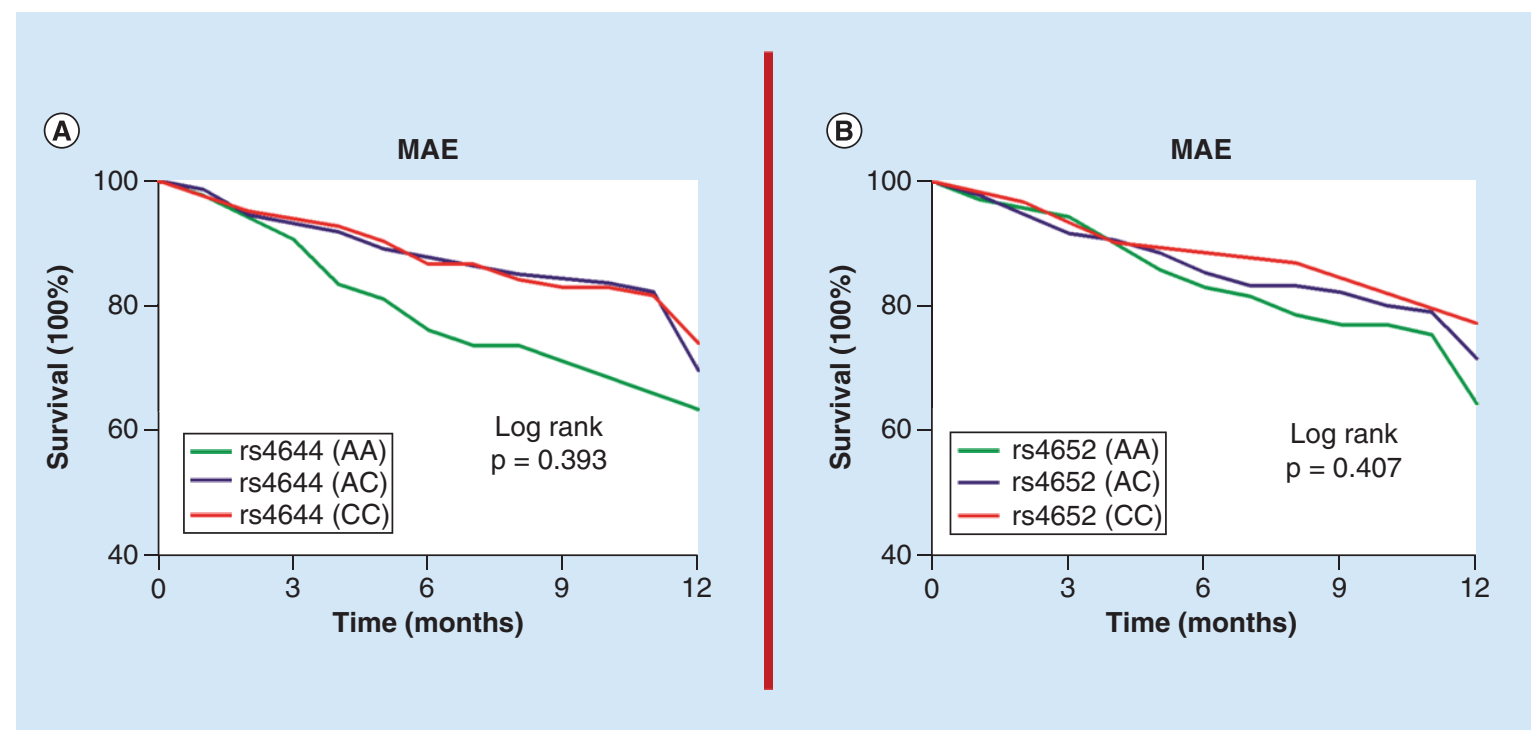

Figure 3. Majors adverse event categorized by the LGALS3 single-nucleotide polymorphisms. During 1 year of follow-up, the Kaplan-Meier survival curves showed that MAEs categorized by the genotypes (AA, AC and CC) of the LGALS3 SNPs at rs4644 (A) and rs4652 (B) loci were not significantly different $(\log -$ rank $p=0.393 ; \log -r a n k p=0.407$; respectively).

MAE: Major adverse event; SNP: Single-nucleotide polymorphism.

\section{Gal-3 \& MF}

There were 198 patients (99\%) with left ventricular LGE and two (1\%) without left ventricular LGE. The LGE patterns were endomyocardial in 90 (45\%) patients, epicardial in 28 (14\%) patients, mid-myocardial in 48 (24\%) patients and transmural in $32(16 \%)$ patients. The plasma Gal-3 concentration at hospitalization was significantly related to the severity of MF $(r=0.808 ; p<0.001)$, with a $65.2 \%$ variation of MF able to be explained by the plasma Gal-3 level $\left(\mathrm{r}^{2}=0.652 ; \mathrm{p}<0.001\right.$; Figure 4). 
Figure 4. Association between plasma galectin-3 level and severity of myocardial fibrosis. Due to the non-normality distribution, plasma Gal-3 and MF were log-transformed to InGal-3 and $\ln \left(\mathrm{V}_{\mathrm{LGE}} / \mathrm{V}_{\mathrm{t}} \%+1\right)$ for linear regression analysis, which indicated that a $65.2 \%$ variation of MF could be explained by plasma Gal-3 $\left(r^{2}=0.652, p<0.001\right)$.

Gal-3: Galectin-3; LGE: Late gadolinium enhancement; MF: Myocardial fibrosis; $V_{L G E}$ : LGE positive volume; $V_{t}$ : Total left ventricle myocardial volume.
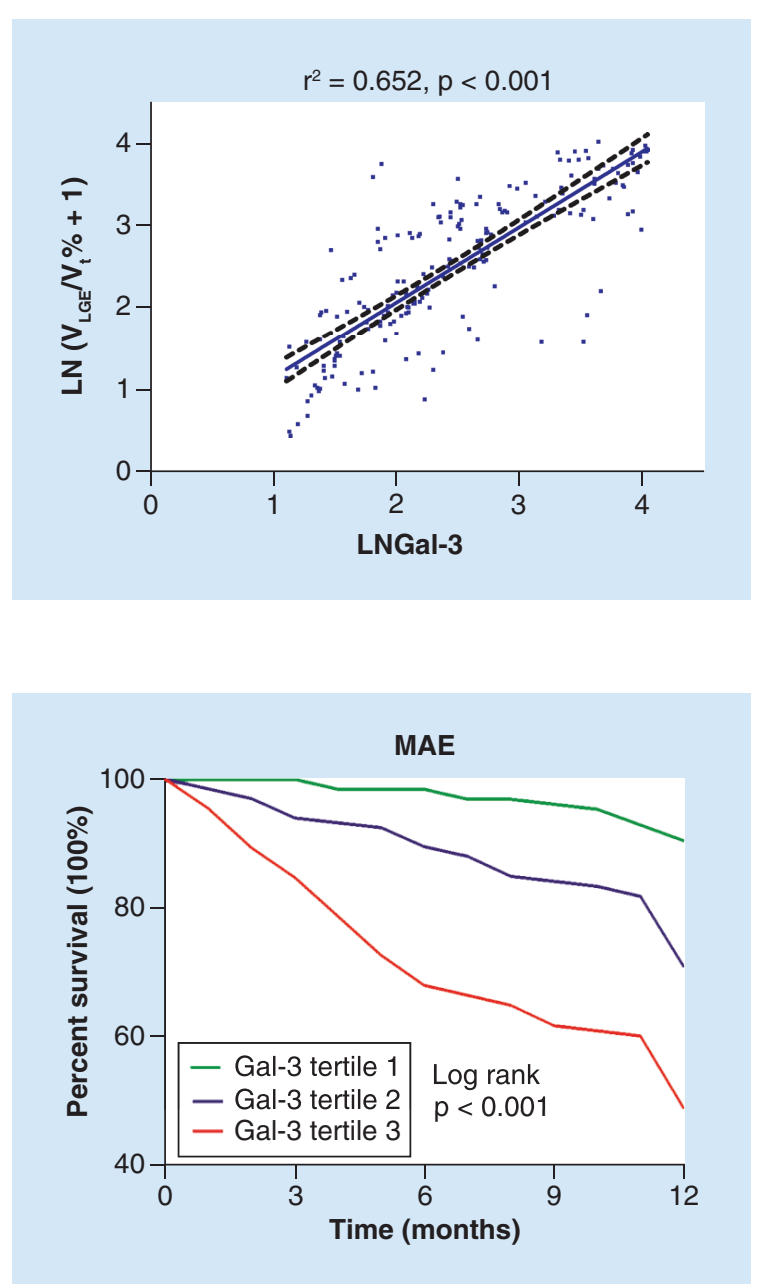

Figure 5. Major adverse events categorized by plasma galectin-3 tertiles. Kaplan-Meier survival curves showed that 1 year cumulative event-free survival decreased with plasma Gal-3 tertiles (log rank $p<0.001$ ).

Gal-3: Galectin-3; MAE: Major adverse event.

\section{Gal-3, MF \& MAE}

Based on the tertiles of the plasma Gal-3 level, we divided study populations into three groups (3.04-7.19, 7.214.67 and $14.68-56.59 \mathrm{ng} / \mathrm{ml}$, respectively) [13]. The association between plasma Gal-3 level and the cumulative event-free survival was evaluated by the Kaplan-Meier survival curves plotted within each tertile of Gal-3. Results revealed that the higher the plasma Gal-3 level, the worse the outcome (log-rank $\mathrm{p}<0.001$; Figure 5). All variables (except allelic frequency and LVEF on cardiac MRI) in Tables $1 \& 2$ were included in a univariate Cox regression analysis, which indicated that the plasma Gal-3 level was significantly related to the prevalence of MAE (hazard ratio [HR]: 1.067; 95\% CI: 1.050-1.083; $\mathrm{p}<0.001$ ). In a multivariable model adjusting for the variables that were significant in the univariate analysis, plasma Gal-3 remained significantly associated with an increased risk of MAEs (HR: 1.057; 95\% CI: 1.034-1.081; $\mathrm{p}<0.001$ ), same as MF (HR: 1.029; 95\% CI: 1.004-1.055; $\mathrm{p}=$ 0.023), BNP (HR: 1.001; 95\% CI: 1.001-1.001; $\mathrm{p}<0.001$ ), LVEDD (HR: 1.043; 95\% CI: 1.011-1.077; $\mathrm{p}=$ 0.009 ) and LVEF (HR: 1.062; 95\% CI: 1.006-1.120; $\mathrm{p}=0.029$; Table 3).

A combination of plasma Gal-3 and MF (global Chi-square $=70.234$ ) was better than plasma Gal-3 alone (global Chi-square $=62.181 ; \mathrm{p}=0.005)$ or MF alone (global Chi-square $=60.170 ; \mathrm{p}=0.002)$, in predicting MAE in patients with AHF within 1 year of follow-up.

\section{Discussion}

Although the relationship between Gal-3, its coding gene variations, MF and the outcome of HF have been separately studied, no study has been conducted to investigate the prognosis of HF considering all of these aspects in a single population, especially in the setting of AHF. In the present prospective, observational study conducted in a cohort of AHF patients with reduced ejection fraction, we found that plasma Gal-3 concentration, the severity 
Table 3. Univariate and multivariate Cox regression analysis for major adverse event.

\begin{tabular}{|c|c|c|c|c|c|c|}
\hline \multirow[t]{2}{*}{ Characteristic } & \multicolumn{3}{|c|}{ Univariate analysis } & \multicolumn{3}{|c|}{ Multivariate analysis } \\
\hline & HR & $95 \% \mathrm{Cl}$ & p-value & HR & $95 \% \mathrm{Cl}$ & p-value \\
\hline Age & 1.021 & $1.002-1.040$ & 0.027 & - & - & 0.656 \\
\hline CKD & 2.392 & $1.290-4.436$ & 0.006 & - & - & 0.101 \\
\hline IV vasoconstrictors & 2.683 & $0.838-8.594$ & 0.097 & - & - & 0.533 \\
\hline IV vasodilators & 1.691 & $1.010-2.830$ & 0.046 & - & - & 0.114 \\
\hline ACEI/ARB & 0.575 & $0.305-1.086$ & 0.088 & - & - & 0.584 \\
\hline Heart rate & 0.984 & $0.968-0.999$ & 0.041 & - & - & 0.701 \\
\hline $\mathrm{BNP}^{\dagger}$ & 1.001 & $1.000-1.001$ & $<0.001$ & 1.001 & $1.001-1.001$ & $<0.001$ \\
\hline sST2 & 1.046 & $1.024-1.068$ & $<0.001$ & - & - & 0.051 \\
\hline eGFR & 0.982 & $0.972-0.993$ & 0.001 & - & - & 0.447 \\
\hline Natrium & 0.922 & $0.868-0.979$ & 0.008 & - & - & 0.612 \\
\hline Hemoglobin & 0.985 & $0.973-0.996$ & 0.008 & - & - & 0.315 \\
\hline LVEDD $^{\dagger}$ & 1.047 & $1.014-1.081$ & 0.004 & 1.043 & $1.011-1.077$ & 0.009 \\
\hline $\operatorname{LVEF}^{\dagger}$ & 0.961 & $0.922-1.001$ & 0.057 & 1.062 & $1.006-1.120$ & 0.029 \\
\hline NYHA class at discharge & 1.579 & $0.943-2.646$ & 0.083 & - & - & 0.336 \\
\hline Gal-3 ${ }^{\dagger}$ & 1.067 & $1.050-1.083$ & $<0.001$ & 1.057 & $1.034-1.081$ & $<0.001$ \\
\hline$M F, V_{L G E} / V_{t} \%^{\dagger}$ & 1.067 & $1.050-1.084$ & $<0.001$ & 1.029 & $1.004-1.055$ & 0.023 \\
\hline \multicolumn{7}{|c|}{ 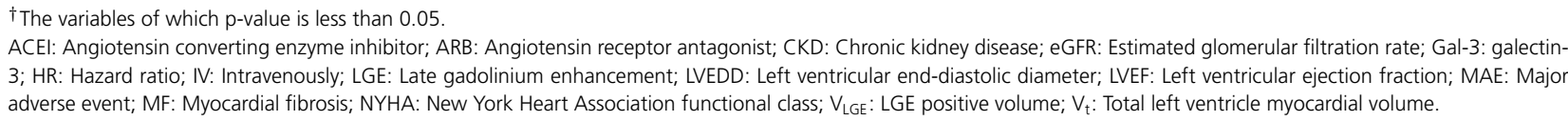 } \\
\hline
\end{tabular}

of left ventricular MF, baseline BNP, LVEDD and LVEF but not the polymorphisms of Gal-3-coding gene were associated with MAEs during 1 year of follow-up.

Gal-3 is involved in many biological processes including MF; the principal pathophysiological basis of HF. Hence, this biomarker was firstly used as a predictor of the outcome in patients with chronic, congestive HF because series measurements of Gal-3 could reflect the development of MF [14]. Then, studies were performed to see whether a single measurement of Gal-3 at/after an attack of AHF could have any prognostic value, however, the results were controversial. The PRIDE study enrolling 599 patients with AHF indicated that an ascended plasma Gal-3 level was the best biomarker to predict 60-day mortality or the composite outcome of death and relapsed HF within 60 days [15,16]. Mueller and colleagues investigated 251 patients with AHF and found Gal-3 to be useful in predicting 1-year all-cause mortality [17]. This association between Gla-3 and AHF prognosis was 'concentrationdependent' that is to say the higher the plasma Gal-3 level, the worse the outcome and vice versa. In an analysis of three clinical trials enrolling 902 patients with AHF, it was suggested that the risk of re-hospitalization for HF was significantly increased once the plasma Gal-3 level exceeded $17.8 \mathrm{ng} / \mathrm{ml}$ [5]. A level lower than the tenth percentile cut-off value of all Gal-3 levels studied in the $\mathrm{COACH}$ study after an occurrence of AHF, identified patients at a low or at no risk of 30- and 180-day mortality and re-hospitalization [18]. Our findings are consistent with these studies. In the present study, we found that plasma Gal-3 closely related to the combination of all-cause mortality and re-hospitalization for HF, and that the higher the plasma Gal-3 level at admission, the lower the cumulative event-free survival during 1 year of follow-up. However, two recent studies have argued against this viewpoint. Other researchers analyzed data from the RELAX-AHF trial and the PROTECT trial where 3194 patients were included, both studies suggested that a single time point-based measurement of Gal-3 could not be used as a standalone predictor of prognosis in the setting of AHF [19,20]. Explanations of these conflicting results included different sample sizes and variable follow-up times. It is crucial to point out that when looking at the relationship between Gal-3 and AHF outcome, one usually assumes that the change in plasma Gal-3 level was in parallel with the presence and severity of MF. Unfortunately, the quantitative assessment of MF was either unavailable or not directly comparable among the aforementioned studies.

On the contrary, our study offers substantial evidence that, in the setting of AHF, the one-time measuring plasma Gal-3 level was linearly correlated with MF detected by cardiac MRI, in other words, the $65.2 \%$ variation of MF could be explained by the plasma Gal-3 level. MF is caused by excessive fibrillary collagen synthesis and deposition with decreased degradation, in which Gal-3 plays a crucial role. MF is the essential pathophysiological mechanism 
of ventricular remodeling, which runs through the entire course of HF. In patients with ischemic or nonischemic cardiomyopathy, it was found that the higher the plasma Gal-3 level, the greater the fibrotic myocardium [21,22]. Furthermore, MF was verified to be a strong predictor for mortality, re-hospitalization or heart transplantation requirement for patients with HF [23,24]. Our study verified that this fact also applied to those presenting with AHF. Therefore, it is reasonable to use the single test of Gal-3 concentration at admission as a surrogate of MF, in the scenario of AHF and to estimate the risk of MAEs in this population. However, this concept warrants further investigation. We also found that, when combining plasma Gal-3 with MF, the power of predicting the prognosis for AHF was strengthened and is higher than that of plasma Gal-3 or MF alone. Although these two factors were found to be correlated in our study, each could have contributed to the observed MAEs through independent pathways in the AHF population. For instance, Gal-3 accelerates the progression of HF resorting to the mechanisms of inflammation [25] and apoptosis [26]. This could explain why the combination of plasma Gal-3 and MF exerts an add-on predictive power.

The LVEDD and LVEF reflect left ventricular remodeling and systolic function, respectively. Researchers found that low LVEF predicted a higher risk of death in patients with AHF [27,28]. Our study showed similar results that in addition to Gal-3, MF and BNP, LVEDD and LVEF were also significant predictors of MAEs in AHF patients with reduced ejection fraction. AHF with preserved ejection fraction could be an important confounder, but its etiology, pathology and pathophysiology are different from that of acute decompensated HF with reduced ejection fraction. We did not include these patients in the present study, but it is worthwhile to address this issue in another study.

ST2 is the receptor for IL-33 of which the soluble and circulating form is sST2. The sST2 is also a very important biomarker in HF. A meta-analysis showed that SST2 predicted the composite outcome of all-cause mortality or HF hospitalization in patients with AHF [29]. We selected this biomarker as a potential predictor of prognosis in AHF. In our study, the plasma sST2 level was higher in MAE group than those in nonevent group. However, in the multivariate regression model, the relationship vanished. This was probably due to the overlapped pathophysiologic role of sST2 and Gal-3 in HF.

While the relationship between Gal-3 and MF was established in the present study, other investigations have suggested that Gal-3 could be the upstream cause of MF rather than a downstream marker [4,7]. An animal experiment showed that the degree of MF and ventricular remodeling increased significantly after the injection of Gal-3 in the pericardial cavity of rats [7]. Therefore, an increase in the level of Gal-3 for any reason should, theoretically, result in the progression of MF and subsequently contribute to a worse outcome among HF patients. In the human genome, Gal-3 is coded by LGALS3 gene. A genome-wide association study identified two nonsynonymous SNPs (rs4644 and rs4652) that are strongly associated with the Gal-3 level in the general population [9]. It was expected that AHF patients who carry SNP variants at these loci would present with variable Gal-3 concentrations, and those with higher Gal-3 levels should show more severe MF and more MAEs during follow-up. Surprisingly, the results of our study indicated that different genotypes of these two LGALS3 gene loci (that is, AA, AC and CC of rs 4644 and rs4652, respectively) were not associated with the variable plasma Gal-3 level, the fibrotic myocardial mass or the MAEs. In fact, a previous observation revealed a similar trend. Zhang et al. found that genetic variation at the rs 4644 and rs 4652 loci of the LGALS3 gene might be related to the susceptibility of dilated cardiomyopathy, but not with patients' plasma Gal-3 levels and mortality [30]. A few considerations need to be taken into account when interpreting these findings. First, the aforementioned genome-wide association study tested individuals are from northern Europe, while both Zhang et al.'s research and our study enrolled Chinese patients. Second, genetic variation is not the only determinant of circulating Gal-3. Different regulatory factors may exist in different races or diseases.

\section{Study limitation}

A few limitations of our study should be mentioned. First, a relatively small number of patients from a single center were followed up with during a short period time. However, the prospective nature and using MAE as the primary end point secured convincing evidence. Second, we were unable to analyze Gal-3 concentration and LGALS3 gene expression at the tissue level in the myocardium. Third, we did not collect data of Gal-3 during follow-up. Although a one-time measurement of Gal-3 is sufficient to predict the outcome as indicated by our results, exploring the fluctuation of plasma Gal-3 concentrations during the acute and chronic phases of HF in the same individual would help to better understand the metabolism of this unique galectin. Finally, we did not perform the cardiac MRI T1-mapping sequence, which can more accurately evaluate myocardial interstitial fibrosis. 


\section{Conclusion}

In patients with AHF, a single time point-based measurement of plasma Gal-3 concentration and the severity of MF are both predictors for the composite outcome of all-cause mortality and re-hospitalization at 1 year of follow-up. However, no association is found, at least in this Chinese AHF patient cohort, between the polymorphisms of the Gal-3-coding gene and plasma Gal-3 concentration, degree of MF or MAEs.

\section{Summary points}

- In acute heart failure (AHF), those with higher plasma Gal-3 level or more severe myocardial fibrosis have worse outcomes.

- In AHF, the higher the plasma Gal-3 levels were, the more severe the myocardial fibrosis was.

- Polymorphisms of LGALS3 are not a significant predictor for the prognosis of AHF.

\section{Author contributions}

$\mathrm{R}$ Bai and H Fu contribute to conception, design, analysis and interpretation of data, drafting and revising the manuscript, and final approval of the manuscript. S Nie and P Luo contribute to conception and design. Y Ruan, Z Zhang and $\mathrm{H}$ Miao contribute to analysis and interpretation of data. X Li and S Wen contribute to revising the manuscript critically for important contents.

\section{Financial \& competing interests disclosure}

This research was supported by the National Key Research and Development Program of China (R. B. 2018YFC1312501); the National Science Foundation of China (SW 81500254 and 81870243; RB 81770322). The authors have no other relevant affiliations or financial involvement with any organization or entity with a financial interest in or financial conflict with the subject matter or materials discussed in the manuscript apart from those disclosed.

\section{Writing assistance}

This manuscript has been copy edited for English by a professional English language service LetPub. This writing assistance was paid for by the authors themselves.

Ethical conduct of research

The Ethics Committee of Beijing Anzhen Hospital approved the protocol of this study, and all patients signed written informed consent. The authors state that they have obtained appropriate institutional review board approval or have followed the principles outlined in the Declaration of Helsinki for all human or animal experimental investigations. In addition, for investigations involving human subjects, informed consent has been obtained from the participants involved.

\section{Open access}

This work is licensed under the Attribution-NonCommercial-NoDerivatives 4.0 Unported License. To view a copy of this license, visit http://creativecommons.org/licenses/by-nc-nd/4.0/

\section{References}

Papers of special note have been highlighted as: $\bullet$ of interest; $\bullet \bullet$ of considerable interest

1. Zhang $\mathrm{Y}$, Zhang J, Butler $\mathrm{J}$ et al. Contemporary epidemiology, management, and outcomes of patients hospitalized for heart failure in China: results from the China heart failure (China-HF) registry. J. Card. Fail. 23(12), 868-875 (2017).

2. Donkor A, Cleland J, McDonagh T, Hardman S. National Heart Failure Audit 2016. 11.07. (2016). https://www.nicor.org.uk/wp-content/uploads/2018/11/Heart-Failure-Summary-Report-2016-17.pdf

3. Calvier L, Miana M, Reboul P et al. Galectin-3 mediates aldosterone-induced vascular fibrosis. Arterioscler. Thromb. Vasc. Biol. 33(1), 67-75 (2013).

4. Yu L, Ruifrok WP, Meissner M et al. Genetic and pharmacological inhibition of galectin-3 prevents cardiac remodeling by interfering with myocardial fibrogenesis. Circ. Heart Fail. 6(1), 107-117 (2013).

5. Meijers WC, Januzzi JL, deFilippi $C$ et al. Elevated plasma galectin-3 is associated with near-term rehospitalization in heart failure: a pooled analysis of 3 clinical trials. Am. Heart J. 167(6), 853-860 (2014).

6. van der Velde AR, Gullestad L, Ueland T et al. Prognostic value of changes in galectin-3 levels over time in patients with heart failure: data from CORONA and COACH. Circ. Heart Fail. 6(2), 219-226 (2013).

- Shows that galectin-3 (Gal-3) offered significant prognostic value for adverse outcomes in patients with heart failure (HF). 
7. Sharma UC, Pokharel S, van Brakel TJ et al. Galectin-3 marks activated macrophages in failure-prone hypertrophied hearts and contributes to cardiac dysfunction. Circulation 110(19), 3121-3128 (2004).

8. Raimond J, Zimonjic DB, Mignon C et al. Mapping of the galectin-3 gene (LGALS3) to human chromosome 14 at region 14q21-22. Mamm. Genome 8(9), 706-707 (1997).

9. de Boer RA, Verweij N, van Veldhuisen DJ et al. A genome-wide association study of circulating galectin-3. PLoS ONE 7(10), e47385 (2012).

-. Indicates that two LGALS3 gene single nucleotide polymorphisms (rs4644 and rs4652) are associated with changes in protein levels and may alter the circulating Gal-3 content.

10. Senni M, Tribouilloy CM, Rodeheffer RJ et al. Congestive heart failure in the community: a study of all incident cases in Olmsted County, Minnesota, in 1991. Circulation 98(21), 2282-2289 (1998).

- $\quad$ Provide us diagnostic criteria for HF.

11. Agoston-Coldea1 L, Lupu S, Petrovai D, Mocan T, Mousseaux E. Correlations between echocardiographic parameters of right ventricular dysfunction and Galectin-3 in patients with chronic obstructive pulmonary disease and pulmonary hypertension. Med. Ultrason. 17(4), 487-495 (2015).

12. Levey AS, Bosch JP, Lewis JB, Greene T, Rogers N, Roth D. A more accurate method to estimate glomerular filtration rate from serum creatinine: a new prediction equation. Modification of Diet in Renal Disease Study Group. Ann. Intern. Med. 130(6), 461-470 (1999).

13. Ho JE, Liu C, Lyass A et al. Galectin-3, a marker of cardiac fibrosis, predicts incident heart failure in the community. J. Am. Coll. Cardiol. 60(14), 1249-1256 (2012).

-. Indicates us how to assess the relationship between Gal-3 level and the cumulative event-free survival.

14. Besler C, Lang D, Urban D et al. Plasma and cardiac Galectin-3 in patients with heart failure reflects both inflammation and fibrosis: implications for its use as a biomarker. Circ. Heart Fail. 10(3), pii: e003804 (2017).

15. Januzzi JL Jr, Camago CA, Anwaruddin S et al. The N-ternimal Pro-BNP investigation of dyspnea in the emergency department (PRIDE) study. Am. J. Cardiol. 95(8), 948-954 (2005).

16. van Kimmenade RR, Januzzi JL Jr, Ellinor PT et al. Utility of amino-terminal pro-brain natriuretic peptide, galectin-3, and apelin for the evaluation of patients with acute heart failure. J. Am. Coll. Cardiol. 48(6), 1217-1224 (2006).

17. Mueller T, Gegenhuber A, Leitner I, Poelz W, Haltmayer M, Dieplinger B. Diagnostic and prognostic accuracy of galectin-3 and soluble ST2 for acute heart failure. Clin. Chim. Acta 463, 158-164 (2016).

- Shows the similar results about relationship between Gal-3 and prognosis of patients with acute heart failure to those in our study.

18. Meijers WC, de Boer RA, van Veldhuisen DJ et al. Biomarkers and low risk in heart failure. Data from COACH and TRIUMPH. Eur. J. Heart Fail. 17(12), 1271-1282 (2015).

19. Demissei BG, Cotter G, Prescott MF et al. A multimarker multi-time point-based risk stratification strategy in acute heart failure: results from the RELAX-AHF trial. Eur. J. Heart Fail. 19(8), 1001-1010 (2017).

20. Demissei BG, Valente MA, Cleland JG et al. Optimizing clinical use of biomarkers in high-risk acute heart failure patients. Eur. J. Heart Fail. 18(3), 269-280 (2016).

21. Lepojärvi ES, Piira OP, Pääkkö E et al. Serum PINP, PIIINP, galectin-3, and ST2 as surrogates of myocardial fibrosis and echocardiographic left ventricular diastolic filling properties. Front. Physiol. 6, 200 (2015).

- Indicates Gal-3 was correlated with myocardial fibrosis.

22. Wu CK, Su MM, Wu YF, Hwang JJ, Lin LY. Combination of plasma biomarkers and clinical data for the detection of myocardial fibrosis or aggravation of heart failure symptoms in heart failure with preserved ejection fraction patients. J. Clin. Med. 7(11), 427 (2018).

23. Cheong BY, Muthupillai R, Wilson JM et al. Prognostic significance of delayed-enhancement magnetic resonance imaging survival of 857 patients with and without left ventricular dysfunction. Circulation 120(21), 2069-2076 (2009).

24. Liu T, Ma X, Liu W et al. Late gadolinium enhancement amount as an independent risk factor for the incidence of adverse cardiovascular events in patients with stage C or D heart failure. Front. Physiol. 7, 484 (2016).

- Shows that myocardial fibrosis was verified to be a strong predictor of adverse cardiovascular events for patients with HF.

25. Frangogiannis NG. The immune system and cardiac repair. Pharmacol. Res. 58(2), 88-111 (2008).

26. Wright RD, Souza PR, Flak MB, Thedchanamoorthy P, Norling LV, Cooper D. Galectin-3-null mice display defective neutrophil clearance during acute inflammation. J. Leukoc. Biol. 101(3), 717-726 (2017).

27. Toma M, Ezekowitz JA, Bakal JA et al. The relationship between left ventricular ejection fraction and mortality in patients with acute heart failure: insights from the ASCEND-HF trial. Eur. J. Heart Fail. 16(3), 334-341 (2014).

28. Adamopoulos C, Zannad F, Fay R et al. Ejection fraction and blood pressure are important and interactive predictors of 4-week mortality in severe acute heart failure. Eur. J. Heart Fail. 9(9), 935-941 (2007).

29. Aimo A, Vergaro G, Ripoli A et al. Meta-analysis of soluble suppression of tumorigenicity-2 and prognosis in acute heart failure. JACC Heart Fail. 5(4), 287-296 (2017). 
30. Zhang Y, Wang Y, Zhai M et al. Influence of LGALS3 gene polymorphisms on susceptibility and prognosis of dilated cardiomyopathy in a northern Han Chinese population. Gene 642, 293-298 (2018).

-. Shows the similar results with those in our study that genetic variation at the rs4644 and rs4652 loci of LGALS3 gene were not related with patients' plasma Gal-3 levels and mortality. 